\title{
Author Correction: Atomically dispersed antimony on carbon nitride for the artificial photosynthesis of hydrogen peroxide
}

Zhenyuan Teng, Qitao Zhang, Hongbin Yang, Kosaku Kato (D), Wenjuan Yang, Ying-Rui Lu, Sixiao Liu, Chengyin Wang (iD, Akira Yamakata (D), Chenliang Su Di , Bin Liu (D) and Teruhisa Ohno (D)

Correction to: Nature Catalysis https://doi.org/10.1038/s41929-021-00605-1, published online 21 May 2021.

In the version of this Article originally published, the Acknowledgements section incorrectly acknowledged funding from the 'Singapore Ministry of Education Academic Research Fund (AcRF) Tier 1 RG115/17 and RG115/18, and Tier 2 MOE2016-T2-2-004, and the Singapore Energy Center (SgEC) grant no. M4062755.120'; this has been changed to the 'Singapore Ministry of Education (Tier 1: RG4/20 and Tier 2: MOET2EP10120-0002) and the Agency for Science, Technology and Research (A^Star IRG: A20E5c0080)'.

Published online: 2 June 2021

https://doi.org/10.1038/s41929-021-00644-8

(C) The Author(s), under exclusive licence to Springer Nature Limited 2021 